\title{
Minimizing CYP2C9 Inhibition of Exposed-Pyridine NAMPT (Nicotinamide Phosphoribosyltransferase) Inhibitors
}

Mark Zak ${ }^{\mathrm{a},{ }^{*}, \text { Po-wai Yuen }}{ }^{\mathrm{c}}$, Xiongcai Liu ${ }^{\mathrm{c}}$, Snahel Patel ${ }^{\mathrm{a}}$, Deepak Sampath ${ }^{\mathrm{a}}$, Jason Oeh ${ }^{\mathrm{a}}$, Bianca M. Liederer ${ }^{\mathrm{a}}$, Weiru Wang ${ }^{\mathrm{a}}$, Thomas O’Brien ${ }^{\mathrm{a}}$, Yang Xiao ${ }^{\mathrm{a}}$, Nicholas Skelton ${ }^{\mathrm{a}}$, Rongbao Hua ${ }^{\mathrm{c}}$, Jasleen Sodhi ${ }^{\mathrm{a}}$, Yunli Wang ${ }^{\mathrm{c}}$, Lei Zhang ${ }^{\mathrm{c}}$, Guiling Zhao ${ }^{\mathrm{a}}$, Xiaozhang Zheng ${ }^{\mathrm{b}}$, Yen-Ching Ho ${ }^{\mathrm{b}}$, Kenneth W. Bair ${ }^{\mathrm{b}}$, Peter S. Dragovich ${ }^{\mathrm{a}}$

${ }^{a}$ Genentech Inc., 1 DNA Way, South San Francisco, California 94080, USA

${ }^{\mathrm{b}}$ FORMA Therapeutics Inc., 500 Arsenal Street, Watertown, Massachusetts 02472, USA

${ }^{c}$ Pharmaron Beijing Co. Ltd., 6 Taihe Road, BDA, Beijing 100176, PR China

* Corresponding Author. E-mail address: mzak@gene.com. Phone: 650-467-4533

\section{Table of Contents}

Title page

LCMS and SFC Methods

Mouse xenograft studies

Crystallography

Measured $\log \mathrm{D}_{7.4}$ method

Coefficients of variation in NAMPT biochemical and cell-based assays

Fsp3 distribution of 119-membered library
S1

$\mathrm{S} 2-\mathrm{S} 17$

S18

S19-S20

$\mathrm{S} 21$

$\mathrm{S} 21-\mathrm{S} 24$

$\mathrm{S} 25$ 


\section{LCMS Methods:}

Method A

Instrument Information: SHIMADZU LCMS-2010EV

LC Parameters:

Column: Shim-pack XR-ODS, $2.2 \mu \mathrm{m}, 3.0 * 50 \mathrm{~mm}$

Mobile Phase A: Water/0.05\%TFA

Mobile Phase B: Acetonitrile

Gradient: $5 \%$ to $100 \% \mathrm{~B}$ in 2.0 minutes, $100 \% \mathrm{~B}$ for 1.1 minutes, $100 \%$ to $5 \% \mathrm{~B}$ in 0.2 minutes, then stop.

Flow Rate: $1.0 \mathrm{~mL} / \mathrm{min}$

Column Temperature: $40^{\circ} \mathrm{C}$

Detector: $254 \mathrm{~nm}$ and ELSD

Sample Preparation: $1 \mathrm{mg} / \mathrm{mL}$ in Methanol

Injection Volume: $1 \mu \mathrm{L}$

Report: Area Normalized Purity

MS Parameters:

Interface: ESI (Positive)

Scan Range: 90-900(m/z)

Method B

Instrument Information: SHIMADZU LCMS-2010EV

LC Parameters:

Column: Waters XBridge C18, $3.5 \mu \mathrm{m}, 3.0 * 50 \mathrm{~mm}$,

Mobile Phase A: Water / 5mM Ammonium Acetate

Mobile Phase B: Methanol

Gradient: $10 \%$ to $70 \%$ B in 3.0 minutes, $70 \%$ to $90 \%$ B in 0.8 minutes, $90 \% \mathrm{~B}$ for 1.7 minutes, $90 \%$ to $10 \% \mathrm{~B}$ in 0.2 minutes, 
then stop.

Flow Rate: $0.9 \mathrm{~mL} / \mathrm{min}$

Column Temperature: $40^{\circ} \mathrm{C}$

Detector: PDA and ELSD

Sample Preparation: $1 \mathrm{mg} / \mathrm{mL}$ in Methanol

Injection Volume: $1 \mu \mathrm{L}$

Report: Area Normalized Purity

MS Parameters:

Interface: ESI (Positive \& Negative)

Scan Range: 90-900 (m/z)

Method C

Instrument Information: SHIMADZU LCMS-2010EV

LC Parameters:

Column: Waters XBridge C18, $3.5 \mu \mathrm{m}, 3.0 * 50 \mathrm{~mm}$,

Mobile Phase A: Water / 5mM Ammonium Acetate

Mobile Phase B: Methanol

Gradient: $10 \%$ to $100 \% \mathrm{~B}$ in 1.8 minutes, $100 \% \mathrm{~B}$ for 1.7 minutes, $100 \%$ to $10 \% \mathrm{~B}$ in 0.1 minutes, then stop.

Flow Rate: $0.9 \mathrm{~mL} / \mathrm{min}$

Column Temperature: $40^{\circ} \mathrm{C}$

Detector: PDA and ELSD

Sample Preparation: $1 \mathrm{mg} / \mathrm{mL}$ in Methanol

Injection Volume: $1 \mu \mathrm{L}$

Report: Area Normalized Purity

MS Parameters:

Interface: ESI (Positive \& Negative)

Scan Range: 90-900 (m/z) 
Instrument Information: SHIMADZU LCMS-2010EV

LC Parameters:

Column: Shim-pack XR-ODS, $2.2 \mu \mathrm{m}, 3.0 * 50 \mathrm{~mm}$

Mobile Phase A: Water/0.05\%TFA

Mobile Phase B: Acetonitrile/0.05\%TFA

Gradient: $5 \%$ to $100 \% \mathrm{~B}$ in 2.0 minutes, $100 \% \mathrm{~B}$ for 1.1 minutes, $100 \%$ to $5 \% \mathrm{~B}$ in 0.2 minutes, then stop.

Flow Rate: $1.0 \mathrm{~mL} / \mathrm{min}$

Column Temperature: $40^{\circ} \mathrm{C}$

Detector: $254 \mathrm{~nm}$ and ELSD

Sample Preparation: $1 \mathrm{mg} / \mathrm{mL}$ in Methanol

Injection Volume: $1 \mu \mathrm{L}$

Report: Area Normalized Purity

MS Parameters:

Interface: ESI (Positive)

Scan Range: 90-900(m/z)

Method E

Instrument Information: SHIMADZU LCMS-2010EV

LC Parameters:

Column: Shim-pack XR-ODS, $2.2 \mu \mathrm{m}, 3.0 * 50 \mathrm{~mm}$

Mobile Phase A: Water $/ 0.05 \%$ TFA

Mobile Phase B:Mobile Phase B: Acetonitrile/0.05\%TFA

Gradient: $5 \%$ to $70 \% \mathrm{~B}$ in 4.0 minutes, $70 \% \mathrm{~B}$ for 1.0 minutes, $70 \%$ to $5 \% \mathrm{~B}$ in 0.3 minutes, then stop.

Flow Rate: $1.0 \mathrm{~mL} / \mathrm{min}$

Column Temperature: $40^{\circ} \mathrm{C}$ 
Detector: $254 \mathrm{~nm}$ and ELSD

Sample Preparation: $1 \mathrm{mg} / \mathrm{mL}$ in Methanol

Injection Volume: $1 \mu \mathrm{L}$

Report: Area Normalized Purity

MS Parameters:

Interface: ESI (Positive)

Scan Range: 90-900(m/z)

Method F

Instrument Information: SHIMADZU LCMS-2010EV

LC Parameters:

Column: Shim-pack XR-ODS, $2.2 \mu \mathrm{m}, 3.0 * 50 \mathrm{~mm}$

Mobile Phase A: Water/0.05\%TFA

Mobile Phase B:Mobile Phase B: Acetonitrile/0.05\%TFA

Gradient: $5 \%$ to $80 \% \mathrm{~B}$ in 4.0 minutes, $80 \% \mathrm{~B}$ for 1.0 minutes, $80 \%$ to $5 \% \mathrm{~B}$ in 0.3 minutes, then stop.

Flow Rate: $1.0 \mathrm{~mL} / \mathrm{min}$

Column Temperature: $40^{\circ} \mathrm{C}$

Detector: $254 \mathrm{~nm}$ and ELSD

Sample Preparation: $1 \mathrm{mg} / \mathrm{mL}$ in Methanol

Injection Volume: $1 \mu \mathrm{L}$

Report: Area Normalized Purity

MS Parameters:

Interface: ESI (Positive)

Scan Range: 90-900(m/z)

Method G

Instrument: SHIMADZU LC/MS-2010EV 


\section{LC Parameters:}

Column: Waters Xselect C18, $3.5 \mu \mathrm{m}, 3.0 * 50 \mathrm{~mm}$

Mobile Phase A: Water/0.1\% formic acid;

Mobile Phase B: Acetonitrile $/ 0.05 \%$ formic acid

Gradient: $5 \%$ to $80 \%$ B in $3.0 \mathrm{~min}, 80 \%$ B for $1.0 \mathrm{~min}, 80 \%$ to $5 \% \mathrm{~B}$ in $0.2 \mathrm{~min}$, then stop;

Flow Rate: $0.9 \mathrm{~mL} / \mathrm{min}$

Column Temperature: $35^{\circ} \mathrm{C}$

Detector: $254 \mathrm{~nm}$ and ELSD

Sample Preparation: $1 \mathrm{mg} / \mathrm{mL}$ in Methanol

Injection Volume: $1 \mu \mathrm{L}$

MS Parameters:

Interface: ESI (Positive \& Negative)

Scan Range: 90-900 (m/z)

Method H

Instrument Information: SHIMADZU LCMS-2010EV

LC Parameters:

Column: Shim-pack XR-ODS, $2.2 \mu \mathrm{m}, 3.0 * 50 \mathrm{~mm}$

Mobile Phase A: Water / 0.05\%TFA

Mobile Phase B: Acetonitrile

Gradient: $5 \%$ to $100 \% \mathrm{~B}$ in 2.0 minutes, $100 \% \mathrm{~B}$ for 1 minute, $100 \%$ to $5 \% \mathrm{~B}$ in 0.3 minutes, then stop.

Flow Rate: $1.0 \mathrm{~mL} / \mathrm{min}$

Column Temperature: $40^{\circ} \mathrm{C}$

Detector: $254 \mathrm{~nm}$ and ELSD

Sample Preparation: $1 \mathrm{mg} / \mathrm{mL}$ in Methanol

Injection Volume: $1 \mu \mathrm{L}$ 
Report: Area Normalized Purity

MS Parameters:

Interface: ESI (Positive )

Scan Range: $90-900(\mathrm{~m} / \mathrm{z})$

Method I

Instrument: SHIMADZU LC/MS-2020

LC Parameters:

Column: Shim-pack XR-ODS, $2.2 \mu \mathrm{m}, 3.0 * 50 \mathrm{~mm}$

Mobile Phase A: Water $/ 0.05 \%$ TFA

Mobile Phase B: Acetonitrile/0.05\% TFA

Gradient: 5\% B to $100 \%$ B for $2.0 \mathrm{~min}, 100 \%$ B for $1.1 \mathrm{~min}, 100 \%$ B to $5 \%$ in $0.1 \mathrm{~min}$, then stop;

Flow Rate: $1.0 \mathrm{~mL} / \mathrm{min}$

Column Temperature: $40{ }^{\circ} \mathrm{C}$

Detector: UV and ELSD

Sample Preparation: $1 \mathrm{mg} / \mathrm{mL}$ in Methanol

Injection Volume: $1 \mu \mathrm{L}$.

MS Parameters:

Interface: ESI (Positive)

Scan Range: 70-900 (m/z)

\section{Method J}

Instrument: SHIMADZU LC/MS-2020

LC Parameters:

Column: Shim-pavk, $2.2 \mu \mathrm{m}, 3.0 * 50 \mathrm{~mm}$

Mobile Phase A: Water $/ 0.05 \%$ TFA

Mobile Phase B: Acetonitrile/0.05\% TFA

Gradient: $5 \%$ B to $60 \%$ B for $4.0 \mathrm{~min}, 60 \%$ B for $1.1 \mathrm{~min}, 60 \%$ B to $5 \%$ in $0.1 \mathrm{~min}$, then stop; Flow Rate: $1.0 \mathrm{~mL} / \mathrm{min}$ 
Column Temperature: $40{ }^{\circ} \mathrm{C}$

Detector: UV and ELSD

Sample Preparation: $1 \mathrm{mg} / \mathrm{mL}$ in Methanol

Injection Volume: $1 \mu \mathrm{L}$.

MS Parameters:

Interface: ESI (Positive)

Scan Range: 70-900 (m/z)

Method K

Instrument: SHIMADZU LC/MS-2020

LC Parameters:

Column: Shim-pavk, $2.2 \mu \mathrm{m}, 3.0 * 50 \mathrm{~mm}$

Mobile Phase A: Water $/ 0.05 \%$ TFA

Mobile Phase B: Acetonitrile/0.05\% TFA

Gradient: $5 \%$ B to $100 \%$ B for $2.0 \mathrm{~min}, 100 \%$ B for $1.1 \mathrm{~min}, 100 \%$ B to $5 \%$ in $0.1 \mathrm{~min}$, then stop;

Flow Rate: $1.0 \mathrm{~mL} / \mathrm{min}$

Column Temperature: $40^{\circ} \mathrm{C}$

Detector: UV and ELSD

Sample Preparation: $1 \mathrm{mg} / \mathrm{mL}$ in Methanol

Injection Volume: $1 \mu \mathrm{L}$.

MS Parameters:

Interface: ESI (Positive)

Scan Range: 70-900 (m/z)

Method L

Instrument: SHIMADZU LC/MS-2020

LC Parameters:

Column: Shim-pack XR-ODS, $2.2 \mu \mathrm{m}, 3.0 * 50 \mathrm{~mm}$

Mobile Phase A: Water $/ 0.05 \%$ TFA 
Mobile Phase B: Acetonitrile/0.05\% TFA

Gradient: $5 \%$ B to $70 \%$ B for $3.5 \mathrm{~min}, 70 \%$ B for $0.7 \mathrm{~min}, 70 \%$ B to $5 \%$ in $0.1 \mathrm{~min}$, then stop;

Flow Rate: $1.0 \mathrm{~mL} / \mathrm{min}$

Column Temperature: $40{ }^{\circ} \mathrm{C}$

Detector: UV and ELSD

Sample Preparation: $1 \mathrm{mg} / \mathrm{mL}$ in Methanol

Injection Volume: $1 \mu \mathrm{L}$.

MS Parameters:

Interface: ESI (Positive)

Scan Range: 70-900 (m/z)

\section{Method M}

Instrument: SHIMADZU LC/MS-2020EV

LC Parameters:

Column: Shim-pack XR-ODS, $2.2 \mu \mathrm{m}, 3.0 * 50 \mathrm{~mm}$

Mobile Phase A: Water/0.05\% TFA

Mobile Phase B: Acetonitrile

Gradient: $5 \%$ to $100 \%$ B in $2.1 \mathrm{~min}, 100 \%$ B for $0.8 \mathrm{~min}, 100 \%$ to $5 \% \mathrm{~B}$ in $0.1 \mathrm{~min}$, then stop;

Flow Rate: $1.0 \mathrm{~mL} / \mathrm{min}$

Column Temperature: $40{ }^{\circ} \mathrm{C}$

Detector: $254 \mathrm{~nm}$ and ELSD

Sample Preparation: $1 \mathrm{mg} / \mathrm{mL}$ in Acetonitrile

Injection Volume: $1 \mu \mathrm{L}$.

MS Parameters:

Interface: ESI (Positive)

Scan Range: 90-900 (m/z)

Method N 
Instrument: SHIMADZU LCMS-2020

LC Parameters:

Column: Shim-pack XR-ODSIII, $2.2 \mu \mathrm{m}, 2.0 * 50 \mathrm{~mm}$

Mobile Phase A: Water/0.1\%FA

Mobile Phase B: Acetonitrile/0.05\% FA

Gradient: $5 \%$ to $100 \%$ B in $2.0 \mathrm{~min}, 100 \%$ B for $1.1 \mathrm{~min}, 100 \%$ to $5 \%$ B in $0.1 \mathrm{~min}$, then stop;

Flow Rate: $0.7 \mathrm{~mL} / \mathrm{min}$

Column Temperature: $40{ }^{\circ} \mathrm{C}$

Detector: $254 \mathrm{~nm}$ and ELSD

Sample Preparation: $1 \mathrm{mg} / \mathrm{mL}$ in Methanol

Injection Volume: $1 \mu \mathrm{L}$.

MS Parameters:

Interface: ESI (Positive)

Scan Range: 90-900 (m/z)

Method O

Instrument: SHIMADZU LCMS-2020

LC Parameters:

Column: Shim-pack XR-ODS, $2.2 \mu \mathrm{m}, 3.0 * 50 \mathrm{~mm}$

Mobile Phase A: Water $/ 0.05 \%$ TFA

Mobile Phase B: Acetonitrile/0.05\% TFA

Gradient: $5 \%$ B to $100 \%$ B for $2.0 \mathrm{~min}, 100 \%$ B for $1.1 \mathrm{~min}, 100 \%$ B to $5 \%$ in $0.1 \mathrm{~min}$, then stop;

Flow Rate: $1.0 \mathrm{~mL} / \mathrm{min}$

Column Temperature: $40{ }^{\circ} \mathrm{C}$

Detector: $254 \mathrm{~nm}$ and ELSD

Sample Preparation: $1 \mathrm{mg} / \mathrm{mL}$ in Methanol; Injection Volume: $1 \mu \mathrm{L}$.

MS Parameters:

Interface: ESI (Positive) 
Scan Range: 70-900 (m/z)

\section{Method P}

Instrument: SHIMADZU LCMS-2020

LC Parameters:

Column: Sunfire $\mathrm{C}_{18}, 3.5 \mu \mathrm{m}, 3.0^{*} 100 \mathrm{~mm}$

Mobile Phase A: Water $/ 0.05 \%$ TFA

Mobile Phase B: Methanol

Gradient: $40 \%$ B for $15 \mathrm{~min}$

Flow Rate: $0.8 \mathrm{~mL} / \mathrm{min}$

Column Temperature: $40^{\circ} \mathrm{C}$

Detector: $254 \mathrm{~nm}$ and ELSD

Sample Preparation: $1 \mathrm{mg} / \mathrm{mL}$ in Methanol; Injection Volume: $1 \mu \mathrm{L}$.

MS Parameters:

Interface: ESI (Positive)

Scan Range: 70-900 (m/z)

Method Q

Instrument: SHIMADZU LCMS-2020

LC Parameters:

Column: Shim-pack XR-ODS, $2.2 \mu \mathrm{m}, 3.0 * 50 \mathrm{~mm}$

Mobile Phase A: Water $/ 0.05 \%$ TFA

Mobile Phase B: Acetonitrile/0.05\% TFA

Gradient: $5 \%$ B to $70 \%$ B for $2.0 \mathrm{~min}, 70 \%$ B for $1.1 \mathrm{~min}, 70 \%$ B to $5 \%$ in $0.1 \mathrm{~min}$, then stop;

Flow Rate: $1.0 \mathrm{~mL} / \mathrm{min}$

Column Temperature: $40^{\circ} \mathrm{C}$

Detector: $254 \mathrm{~nm}$ and ELSD

Sample Preparation: $1 \mathrm{mg} / \mathrm{mL}$ in Methanol; Injection Volume: $1 \mu \mathrm{L}$. MS Parameters: 
Interface: ESI (Positive)

Scan Range: 70-900 (m/z)

Method R

Instrument: SHIMADZU LCMS-2020

LC Parameters:

Column: Shim-pack XR-ODS, $2.2 \mu \mathrm{m}, 3.0 * 50 \mathrm{~mm}$

Mobile Phase A: Water/0.05\% TFA

Mobile Phase B: Acetonitrile/0.05\% TFA

Gradient: $5 \%$ B to $60 \%$ B for $2.0 \mathrm{~min}, 60 \%$ B for $1.1 \mathrm{~min}, 70 \%$ B to $5 \%$ in $0.1 \mathrm{~min}$, then stop;

Flow Rate: $1.0 \mathrm{~mL} / \mathrm{min}$

Column Temperature: $40{ }^{\circ} \mathrm{C}$

Detector: $254 \mathrm{~nm}$ and ELSD

Sample Preparation: $1 \mathrm{mg} / \mathrm{mL}$ in Methanol; Injection Volume: $1 \mu \mathrm{L}$.

MS Parameters:

Interface: ESI (Positive)

Scan Range: 70-900 (m/z)

\section{Method S}

Instrument: SHIMADZU LCMS-2020

LC Parameters:

Column: Shim-pack XR-ODS, $2.2 \mu \mathrm{m}, 3.0 * 50 \mathrm{~mm}$

Mobile Phase A: Water/0.1\% formic acid

Mobile Phase B: Acetonitrile/0.05\% formic acid

Gradient: $5 \%$ to $100 \% \mathrm{~B}$ in 3.0 minutes, $100 \% \mathrm{~B}$ for 1.5 minutes, $100 \%$ to $5 \% \mathrm{~B}$ in 0.2 minutes, then stop

Flow Rate: $1.0 \mathrm{~mL} / \mathrm{min}$

Column Temperature: $40{ }^{\circ} \mathrm{C}$ 
Detector: $254 \mathrm{~nm}$ and ELSD

Sample Preparation: $1 \mathrm{mg} / \mathrm{mL}$ in Acetonitrile

Injection Volume: $1 \mu \mathrm{L}$

Report: Area Normalized Purity

MS Parameters:

Interface: ESI (Positive)

Scan Range: 90-900 (m/z)

\section{Method T}

Instrument: SHIMADZU LCMS-2020

LC Parameters:

Column: Shim-pack XR-ODS, $2.2 \mu \mathrm{m}, 3.0 * 50 \mathrm{~mm}$

Mobile Phase A: Water/0.1\% formic acid

Mobile Phase B: Acetonitrile/0.05\% formic acid

Gradient: $5 \%$ to $100 \% \mathrm{~B}$ in 2.0 minutes, $100 \% \mathrm{~B}$ for 1.1 minutes, $100 \%$ to $5 \% \mathrm{~B}$ in 0.1 minutes, then stop

Flow Rate: $1.0 \mathrm{~mL} / \mathrm{min}$

Column Temperature: $40{ }^{\circ} \mathrm{C}$

Detector: $254 \mathrm{~nm}$ and ELSD

Sample Preparation: $1 \mathrm{mg} / \mathrm{mL}$ in Acetonitrile

Injection Volume: $1 \mu \mathrm{L}$

Report: Area Normalized Purity

MS Parameters:

Interface: ESI (Positive)

Scan Range: 90-900 (m/z)

Method U

Instrument: SHIMADZU LCMS-2020 


\section{LC Parameters:}

Column: Gemini-NX 3u C18 110A

Mobile Phase A: Water/0.04\% Ammonia

Mobile Phase B: Acetonitrile

Gradient: $5 \%$ to $100 \%$ B in $2.0 \mathrm{~min}, 100 \%$ B for $1.1 \mathrm{~min}, 100 \%$ to $5 \% \mathrm{~B}$ in $0.1 \mathrm{~min}$, then stop;

Flow Rate: $1.0 \mathrm{~mL} / \mathrm{min}$

Column Temperature: $35^{\circ} \mathrm{C}$

Detector: $254 \mathrm{~nm}$ and ELSD

Sample Preparation: $1 \mathrm{mg} / \mathrm{mL}$ in Methanol

Injection Volume: $1 \mu \mathrm{L}$.

MS Parameters:

Interface: ESI (Positive \& Negative)

Scan Range: 90-900 (m/z)

\section{Method V}

Instrument: SHIMADZU LCMS-2020

LC Parameters:

Column: Sunfire C18, $3.5 \mu \mathrm{m}, 3.0 * 50 \mathrm{~mm}$

Mobile Phase A: Water/0.1\% FA

Mobile Phase B: Acetonitrile/0.05\% FA

Gradient: $5 \%$ to $70 \% \mathrm{~B}$ in 4.0 minutes, $70 \% \mathrm{~B}$ for 1.8 minutes, $100 \%$ to $5 \% \mathrm{~B}$ in 0.3 minutes, then stop

Flow Rate: $1.0 \mathrm{~mL} / \mathrm{min}$

Column Temperature: $40{ }^{\circ} \mathrm{C}$

Detector: $254 \mathrm{~nm}$ and ELSD

Sample Preparation: $1 \mathrm{mg} / \mathrm{mL}$ in Acetonitrile

Injection Volume: $1 \mu \mathrm{L}$

Report: Area Normalized Purity 
MS Parameters:

Interface: ESI (Positive)

Scan Range: 90-900 (m/z)

\section{Method W}

Instrument: SHIMADZU LCMS-2020

LC Parameters:

Column: Sunfire C18, $3.5 \mu \mathrm{m}, 3.0 * 50 \mathrm{~mm}$

Mobile Phase A: Water $/ 0.1 \%$ FA

Mobile Phase B: Acetonitrile/0.05\% FA

Gradient: $5 \%$ to $100 \%$ B in 2.0 minutes, $100 \%$ B for 1.1 minutes, $100 \%$ to $5 \% \mathrm{~B}$ in 0.1 minutes, then stop

Flow Rate: $1.0 \mathrm{~mL} / \mathrm{min}$

Column Temperature: $40^{\circ} \mathrm{C}$

Detector: $254 \mathrm{~nm}$ and ELSD

Sample Preparation: $1 \mathrm{mg} / \mathrm{mL}$ in Acetonitrile

Injection Volume: $1 \mu \mathrm{L}$

Report: Area Normalized Purity

MS Parameters:

Interface: ESI (Positive)

Scan Range: 90-900 (m/z)

Method X

Instrument Information: Agilent 1200 LC with Agilent Quadrupole 6140 MS

LC Parameters:

Column: Agilent ZORBAX SB-C18, $1.8 \mu \mathrm{m}, 2.1 * 30 \mathrm{~mm}$

Mobile Phase A: Water with $0.1 \%$ FA

Mobile Phase B: Acetonitrile with 0.1\%FA 
Gradient: $3-95 \%$ B in $7.0 \mathrm{~min}$, then $95 \%$ B for $1.5 \mathrm{~min}$

Flow Rate: $0.4 \mathrm{~mL} / \mathrm{min}$

Column Temperature: $35^{\circ} \mathrm{C}$

Detector: UV, $254 \mathrm{~nm}$

Sample Preparation: $1 \mathrm{mg} / \mathrm{mL}$ in Acetonitrile

Injection Volume: $1 \mu \mathrm{L}$

Report: Area Normalized Purity

MS Parameters:

Ionization: ESI (Positive)

Scan Range: $110-800(\mathrm{~m} / \mathrm{z})$

Method Y

Instrument Information: Waters Acquity UPLC with Waters LCT Premier XE MS

LC Parameters:

Column: Acquity UPLC BEH C18, $1.7 \mu \mathrm{m}, 2.1 * 50 \mathrm{~mm}$

Mobile Phase A: Water with $0.05 \%$ TFA

Mobile Phase B: Acetonitrile with $0.05 \%$ TFA

Gradient: $2 \%-98 \% \mathrm{~B}$ in $6 \mathrm{~min}, 98 \% \mathrm{~B}$ for $1 \mathrm{~min}$

Flow Rate: $0.6 \mathrm{~mL} / \mathrm{min}$

Column Temperature: $40{ }^{\circ} \mathrm{C}$

Detector: UV, $254 \mathrm{~nm}$

MS Parameters:

Ionization: ESI (Positive)

Scan Range: 10-800 (m/z) 


\section{Analytical Chiral SFC Method:}

\section{Method SFC1}

Instrument: Waters UPC2

Column: Chiralpak AD-3 (50 x 4.6mm, $3 \mu \mathrm{m})$

Mobile Phase A: Carbon Dioxide

Mobile Phase B: Methanol with $0.1 \% \mathrm{NH}_{4} \mathrm{OH}$

Method: $5-60 \% \mathrm{~B}$ in $1.8 \mathrm{~min}$

Run time: $2.5 \mathrm{~min}$

Flow: $4 \mathrm{~mL} / \mathrm{min}$

Pressure: 105 bar

Temperature: $40{ }^{\circ} \mathrm{C}$

Detection Wavelength: $220 \mathrm{~nm}$ 


\section{Mouse Xenograft Efficacy Studies}

All in vivo studies were approved by Genentech's Institutional Animal Care and Use Committee (IACUC) and adhere to the NIH Guidelines for the Care and Use of Laboratory Animals. Human HT-1080 fibrosarcoma tumor xenografts were established by subcutaneous injection of 10 million cells suspended in a 1:1 ratio of HBSS and phenol red-free matrigel (Becton Dickinson Bioscience, San Jose, CA) into female NCR nude mice from Taconic (Cambridge City, IN). Animals were distributed into treatment groups ( $\mathrm{n}=10 /$ group) when tumors reached a mean volume of approximately 200-300 $\mathrm{mm}^{3}$. Compound $\mathbf{6 8}$ was administered twice daily (BID) at $25 \mathrm{mg} / \mathrm{kg}$ or $50 \mathrm{mg} / \mathrm{kg}$ by oral gavage (PO) in vehicle $(60 \%$ PEG400 / 10\% EtOH / 30\% $\mathrm{H}_{2} \mathrm{O}$ ). Tumor volumes were determined using digital calipers (Fred V. Fowler Company, Inc., Newton, MA) using the formula $(\mathrm{L} x \mathrm{~W} \times \mathrm{W}) / 2$. Percentage tumor growth (\% TGI) was calculated based on the following formula: $\%$ TGI $=100 \times($ day 21 mean volume of tumors in animals given vehicle - day 21 mean volume of tumors in animals given the test article)/day 21 mean volume of tumors in animals given vehicle. Tumor sizes and body weights were recorded twice weekly over the course of the study. Mice with tumor volumes $\geq 2000 \mathrm{~mm}^{3}$ or with losses in body weight $\geq 20 \%$ from their weight at the start of treatment were euthanized per IACUC guidelines. Statistical significance was defined as $\mathrm{p}<$ 0.05. To compare two groups, an unpaired t-test assuming unequal variances was used. 


\section{Crystal structure determination}

The NAMPT protein was expressed, purified and co-crystallized with inhibitor compound as previously described. ${ }^{i}$ For co-crystallization, the compound (41) was initially dissolved in DMSO at $50 \mathrm{mM}$ as the stock solution. The compound in stock solution was than add to the protein solution at 1:50 ratio to reach final compound concentration of $1 \mathrm{mM}$.

The diffraction data set of $\mathbf{4 1}$ were collected at Shanghai Synchrotron Radiation Facility. The data set was processed with the HKL2000 package (Z. Otwinowski and W. Minor," Processing of X-ray Diffraction Data Collected in Oscillation Mode ", Methods in Enzymology, Volume 276: Macromolecular Crystallography, part A, p.307-326, 1997). Data collection and structure refinement statistics are summarized in Tables S1.

The structure was solved by molecular replacement (MR) with known Nampt struture (PDB code: 4KFO) as the search model using the program Phaser. The structure was further refined using program REFMAC5 (Murshudov GN et al, Acta Crystallogr. D Biol. Crystallogr. 53, 240-255, 1997) and PHENIX (Adams PD et al, Acta Crystallogr. D Biol. Crystallogr. 66, 213-221, 2010) using the maximum likelihood target functions, anisotropic individual B-factor refinement method, and TLS refinement method, to achieve convergence. The data and refinement statistics are shown in Table S1. 
Table S1: X-ray crystallography data and refinement statistics

\begin{tabular}{|c|c|}
\hline Compound & 41 \\
\hline PDB accession code & $5 \mathrm{KIT}$ \\
\hline Space group & $\mathrm{P} 2_{1}$ \\
\hline Unit cell & $\begin{array}{c}a=60.7 \AA, b=106.6 \AA, c=83.3 \AA, \\
\alpha=90^{\circ} \beta=96.7^{\circ} \gamma=90^{\circ}\end{array}$ \\
\hline Resolution & $50-1.60 \AA$ \\
\hline $\begin{array}{l}\text { Total number of } \\
\text { reflections }(\%)\end{array}$ & $131279(9705)^{1}$ \\
\hline Completeness & $94.9(70.0)$ \\
\hline Redundancy & $3.0(2.0)$ \\
\hline $\mathrm{I} / \sigma$ & $10.6(2.7)$ \\
\hline Rsym $^{2}$ & $0.099(0.295)$ \\
\hline Resolution range & $50-1.60 \AA$ \\
\hline Rcryst $^{3} /$ Rfree $^{4}$ & $0.137 / 0.180$ \\
\hline Non-hydrogen atoms & 8832 \\
\hline Water molecules & 1182 \\
\hline Average B (Overall) & $19.89 \AA^{2}$ \\
\hline r.m.s.d. bond lengths & $0.006 \AA$ \\
\hline r.m.s.d. angles & $1.024^{\circ}$ \\
\hline Ramachandran (C/A/G/D) & $0.911 / 0.089 / 0 / 0$ \\
\hline
\end{tabular}

${ }^{1}$ Values in parentheses are of the highest resolution shell $1.66 \AA-1.60 \AA$

${ }^{2} \mathrm{Rsym}=\Sigma\left|\mathrm{I}_{\mathrm{hi}}-\mathrm{I}_{\mathrm{h}}\right| / \Sigma \mathrm{I}_{\mathrm{hi}}$, where $\mathrm{I}_{\mathrm{hi}}$ is the scaled intensity of the $i$ th symmetry-related observation of reflection $h$ and $\mathrm{I}_{\mathrm{h}}$ is the mean value.

${ }^{3}$ Rcryst $=\Sigma_{h}\left|F_{o h}-F_{c h}\right| / \Sigma_{h} F_{o h}$, where $F_{o h}$ and $F_{c h}$ are the observed and calculated structure factor amplitudes for reflection $h$.

${ }^{4}$ Value of Rfree is calculated for $5 \%$ randomly chosen reflections not included in the refinement. 


\section{Measured $\operatorname{LogD} D_{7.4}$ experimental procedure:}

Measured $\log \mathrm{D}$ values at $\mathrm{pH} 7.4\left(\log \mathrm{D}_{7.4}\right)$ were determined as follows: $10 \mathrm{mM}$ DMSO stock solutions of test compounds were prepared. Each DMSO stock was then added to a 1:1 mixture of octanol / aqueous phosphate buffered saline (PBS, $\mathrm{pH}=7.4$ ) to give final DMSO concentrations of $1 \%$. An internal standard was added to each of the testing samples to ensure data quality. The samples were shaken for 1 hour, then the phases were separated and transferred to separate microtiter plates. The concentrations of test compounds in each phase were monitored by a MRM (multiple reaction monitoring) response with a $2 \mathrm{~min}$ LC/MS/MS method on the Sciex API-4000. The $\log \mathrm{D}_{7.4}$ values were calculated as the $\log$ of the signal ratio in the two phases, Log[octanol-MRM response/PBS-MRM response]. 16 commercial compounds were included in every assay run as standard controls. The dynamic range encompassed $\log \mathrm{D}_{7.4}$ values between -0.5 to 5.5 .

Table S2: Coefficients of variation in NAMPT biochemical and cellular inhibition assays.

\begin{tabular}{ccccc}
\hline Cmpd. & $\begin{array}{c}\text { NAMPT } \\
\mathrm{IC}_{50} \\
(\mu \mathrm{M})^{\mathrm{a}}\end{array}$ & $\begin{array}{c}\text { NAMT } \\
\mathrm{IC}_{50} \\
\mathrm{CV}^{\mathrm{b}}\end{array}$ & $\begin{array}{c}\mathrm{A} 2780 \\
\mathrm{IC}_{50} \\
(\mu \mathrm{M})^{\mathrm{c}}\end{array}$ & $\begin{array}{c}\mathrm{A} 2780 \\
\mathrm{IC}_{50} \\
\mathrm{CV}^{\mathrm{d}}\end{array}$ \\
\hline $\mathbf{1}$ & 0.005 & 0.36 & 0.002 & 0.49 \\
$\mathbf{2}$ & 0.007 & 0.69 & 0.032 & 0.16 \\
$\mathbf{3}$ & 0.006 & 0.63 & 0.004 & 0.43 \\
$\mathbf{4}$ & 0.004 & 0.69 & 0.006 & 0.06 \\
$\mathbf{5}$ & 0.011 & 0.81 & 0.011 & 0.38 \\
$\mathbf{6}$ & 0.039 & 0.40 & 0.011 & 0.29 \\
$\mathbf{7}$ & 0.002 & 0.53 & 0.001 & 0.47 \\
$\mathbf{8}$ & 0.003 & 0.28 & 0.001 & 0.10 \\
$\mathbf{9}$ & 0.003 & 0.39 & 0.144 & 0.25 \\
$\mathbf{1 0}$ & 0.059 & 0.15 & $>2$ &
\end{tabular}




\begin{tabular}{|c|c|c|c|c|}
\hline 11 & 0.003 & 0.18 & 0.070 & 0.25 \\
\hline 12 & 0.006 & 0.08 & 0.076 & 0.10 \\
\hline 13 & 0.010 & 0.72 & 0.006 & 0.36 \\
\hline 14 & 0.010 & 0.56 & 0.006 & 0.10 \\
\hline 15 & 0.008 & 0.47 & 0.005 & 0.31 \\
\hline 16 & $0.064^{\mathrm{e}}$ & & 0.058 & 0.17 \\
\hline 17 & 0.014 & 0.82 & 0.015 & 0.23 \\
\hline 18 & 0.074 & 0.66 & 0.378 & 0.07 \\
\hline 19 & 0.015 & 0.54 & 0.009 & 0.54 \\
\hline 20 & $0.098^{\mathrm{e}}$ & & 0.828 & 0.04 \\
\hline 21 & 0.402 & 0.55 & $>2$ & \\
\hline 22 & 0.473 & 0.4 & $>2$ & \\
\hline 23 & $>2$ & & & \\
\hline 24 & $0.021^{\mathrm{e}}$ & & 0.016 & 0.28 \\
\hline 25 & $0.014^{\mathrm{e}}$ & & 0.027 & 0.29 \\
\hline 26 & 0.423 & 1.01 & $>2$ & \\
\hline 27 & $>2$ & & $>2$ & \\
\hline 28 & $>2$ & & $>2$ & \\
\hline 29 & $0.002^{\mathrm{e}}$ & & 0.013 & 0.14 \\
\hline$( \pm)-\mathbf{3 0}$ & 0.830 & 0.28 & & \\
\hline$( \pm)-\mathbf{3 1}$ & 0.074 & 0.13 & 0.101 & 0.24 \\
\hline 32 & $>1.7$ & & & \\
\hline$( \pm)-\mathbf{3 3}$ & $>2$ & & & \\
\hline$( \pm)-34$ & 0.059 & 0.14 & $>2$ & \\
\hline$( \pm)-35$ & $>2$ & & & \\
\hline$( \pm)-36$ & 0.024 & 0.88 & 0.070 & 0.04 \\
\hline$( \pm)-37$ & 0.018 & 0.06 & 0.119 & 0.11 \\
\hline$( \pm)-38$ & 0.009 & 0.22 & 0.203 & 0.12 \\
\hline
\end{tabular}




\begin{tabular}{|c|c|c|c|c|}
\hline$( \pm)-39$ & 0.010 & 0.07 & 0.036 & 0.47 \\
\hline$( \pm)-40$ & 0.0072 & 0.37 & 0.0016 & 0.82 \\
\hline 41 & 0.013 & 0.33 & 0.001 & 0.01 \\
\hline 42 & 0.035 & 0.50 & 0.034 & 0.12 \\
\hline$( \pm)-43$ & 0.0212 & 0.90 & 0.0016 & 0.05 \\
\hline$( \pm)-44$ & 0.0128 & 0.08 & 0.0044 & 0.19 \\
\hline$( \pm)-45$ & 0.0060 & 0.64 & 0.0004 & 0.14 \\
\hline$( \pm)-46$ & 0.0161 & 0.67 & 0.0058 & 0.14 \\
\hline$( \pm)-47$ & 0.0219 & 0.74 & 0.0069 & 0.74 \\
\hline$( \pm)-48$ & 0.0589 & 0.62 & 0.0133 & 0.11 \\
\hline$( \pm)-49$ & 0.0478 & 0.30 & 0.0112 & 0.17 \\
\hline$( \pm)-\mathbf{5 0}$ & 0.0265 & 0.19 & 0.0248 & 0.03 \\
\hline$( \pm)-51$ & 0.0210 & 0.86 & 0.0035 & 0.09 \\
\hline$( \pm)-52$ & 0.0061 & 0.98 & 0.0053 & 0.55 \\
\hline$( \pm)-53$ & $0.0410^{\mathrm{e}}$ & & $0.0312^{\mathrm{e}}$ & \\
\hline$( \pm)-54$ & 0.0142 & 0.06 & 0.0038 & 0.09 \\
\hline$( \pm)-55$ & 0.0274 & 0.04 & 0.0065 & 0.05 \\
\hline$( \pm)-56$ & 0.0204 & 0.63 & 0.0040 & 0.30 \\
\hline$( \pm)-\mathbf{5 7}$ & 0.0086 & 0.85 & 0.0062 & 0.05 \\
\hline$( \pm)-\mathbf{5 8}$ & 0.0412 & 1.13 & 0.0075 & 0.05 \\
\hline$( \pm)-59$ & 0.0044 & 0.13 & 0.0016 & 0.94 \\
\hline$( \pm)-60$ & 0.0481 & 0.14 & 0.0207 & 0.02 \\
\hline$( \pm)-61$ & 0.5554 & 0.84 & 0.7535 & 0.06 \\
\hline$( \pm)-62$ & 0.0151 & 0.24 & 0.0043 & 0.11 \\
\hline$( \pm)-63$ & 0.0387 & 0.54 & 0.0294 & 0.01 \\
\hline$( \pm)-64$ & $0.0222^{\mathrm{e}}$ & & 0.0048 & 0.18 \\
\hline$( \pm)-65$ & 0.0067 & 0.71 & 0.0016 & 0.03 \\
\hline
\end{tabular}




\begin{tabular}{ccccc}
$( \pm)-66$ & 0.0171 & 0.52 & 0.0022 & 0.90 \\
$( \pm)-67$ & $0.0119^{\mathrm{e}}$ & & 0.0010 & 0.10 \\
$\mathbf{6 8}$ & 0.0055 & 0.47 & 0.0006 & 0.11 \\
$\mathbf{6 9}$ & 0.075 & 0.35 & & \\
\hline
\end{tabular}

Unless otherwise indicated, all assay results are reported as the arithmetic mean of at least two separate runs $(\mathrm{n} \geq 2)$.

${ }^{a}$ NAMPT biochemical inhibition.

${ }^{\mathrm{b}} \mathrm{CV}$ (coefficient of variation) in the NAMPT biochemical assay is calculated as follows: $\mathrm{CV}=$ standard deviation $\div$ mean.

${ }^{c}$ Antiproliferation activity determined in cell culture experiments using A2780 cell line. This inhibition can be reversed by addition of $0.33 \mu \mathrm{M}$ of NMN, strongly implicating NAMPT inhibition as the causative MOA.

${ }^{\mathrm{d}} \mathrm{CV}$ (coefficient of variation) in the NAMPT cell-based assay is calculated as follows: $\mathrm{CV}=$ standard deviation $\div$ mean.

${ }^{\mathrm{e}}$ Single determination $(\mathrm{n}=1)$. 


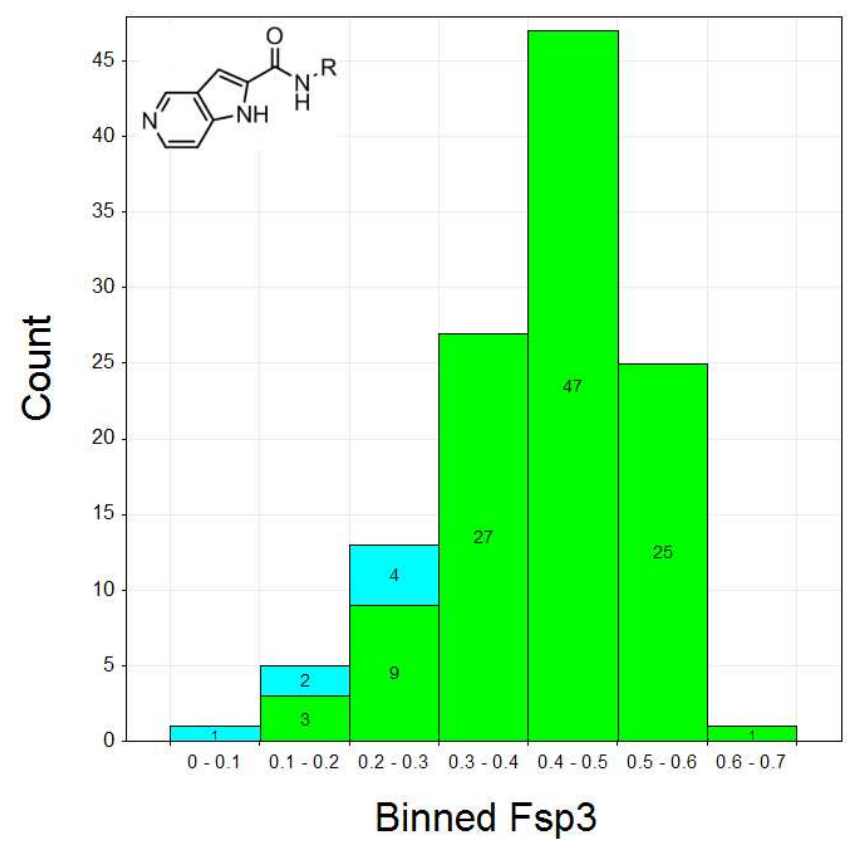

Figure S1. Fsp3 distribution of compounds in 119-membered library. Green denotes the absence of aromatic groups within the "R" substituent of the inhibitors (112/119 compounds). These $R$ moieties were comprised of groups such as aliphatic chains, heteroatoms, saturated cycles, and combinations thereof. Teal denotes the presence of an aromatic group within the " $\mathrm{R}$ " substituent of the inhibitors (7/119 compounds).

'Zheng, X.; Bauer, P.; Baumeister, T.; Buckmelter, A. J.; Caligiuri, M.; Clodfelter, K. H.; Han, B.; Ho, Y.-C.; Kley, N.; Lin, J.; Reynolds, D. J.; Sharma, G.; Smith, C. C.; Wang, Z.; Dragovich, P. S.; Oh, A.; Wang, W.; Zak, M.; Gunzner-Toste, J.; Zhao, G.; Yuen, P.-w.; Bair, K. W. Structure-Based Identification of Ureas as Novel Nicotinamide Phosphoribosyltransferase (Nampt) Inhibitors. J. Med. Chem. 2013, 56, 4921-4937. 